International Journal of Biomedicine I June 2019 - Volume 9, Issue Suppl_1:

Abstracts From the Second Russian International Conference "Cryo-electron microscopy 2019: achievements and prospects"

POSTER ABSTRACT PRESENTATIONS

SESSION TITLE: NEW METHODS OF SAMPLE PREPARATION AND DATA PROCESSING FOR CRYOELECTRON MICROSCOPY

DOI: 10.21103/IJBM.9.Suppl_1.P39

\title{
Abstract P-39: Novel Perspectives for CLEM Techniques in Multiparametric Morphology Protocols
}

Oleg V. Gradov

N.N. Semenov Institute of Chemical Physics, Russian Academy of Sciences, Moscow, Russia

Background: It is well known that CLEM techniques are very informative SEM strategies and approaches based on the fluorescence dyes and pigments as labels and electron microscopy contrast agents in some cases. Despite this fact, the general problem is the impossibility of target-label detection of most physical properties of cells or tissues, which can be visualized using different techniques of standard optical microscopy, because some most important optical microscopy techniques (such as polarizing microscopy, interference-phase contrast, DIC, etc.) and in situ electron microscopy techniques (environmental scanning electron microscopies with different gaseous atmospheres, ballistic electron microscopy, 4D strobe-electron microscopy, etc.) have not been combined within the CLEM's ideology. In the framework of this problem, our group joined the initiative R\&D program on correlated light and electron microscopy development. Our initiative mission was previously focused on the development of databases and knowledge bases for annotations of the possible CLEM approaches without a fluorescence labeling (in situ using ESEM-like systems and in cryo-mode).

Methods: Some novel techniques of electron microscopy for biomedical and physicochemical applications were developed using TRIZ database background and novel techniques of combinatorial rapid prototyping and optimization (know how). For example, it is possible to create some exotic CLEMmicroscopes, such as: Laser Holographic CLEM; Second-Harmonic Imaging CLEM (SHI-CLEM); Interference Reflection CLEM (IRM-CLEM); CLEM Microinterferometry using Linnik Interferometer (without chromatic dispersion or other optical aberrations); Single Plane Illumination CLEM (SPIMCLEM); Nomarski or Pluto Differential Interference Contrast CLEMs (DIC-CLEMs); Phase Contrast and Anoptral Phase Contrast CLEM; Diffraction Imaging CLEM (DICLEM); Rheinberg Illumination CLEM; Hoffmann's Modulation Contrast CLEM; Photo-Activated Localization CLEM (PAL-CLEM or FPALCLEM); Total Internal Reflection Fluorescence CLEM (TIRF-CLEM); Multiplane/Multifocal Plane CLEM (CLEMUM).

Results: In our work the database of such not implemented yet, but rather possible and computed (in our program) variants is described. Some of them were realized in hardware and some of them have a 
technical documentation pull providing prerequisites for multiphysical (COMSOL MULTIPHYSICS assisted) modeling within CAD-CAM-CAE approaches. Among the conventional staining techniques, it is possible to apply the dispersion staining methods in CLEM (both for electron microscopy and for optical one).

Conclusion: Novel techniques for correlation light and electron microscopy (CLEM) are proposed and annotated in this brief communication.

\section{Key Words: CLEM • ESEM • CAD-CAM-CAE • MULTIPHYSICS}

Sources of Funding: This work was supported by FASO (project 0082-2018-0006, registration code AAAA-A18-118020890097-1).

International Journal of Biomedicine. 2019;9 Suppl 1: S34. doi: 10.21103/IJBM.9.Suppl_1.P39

(C)2019 International Medical Research and Development Corporation 